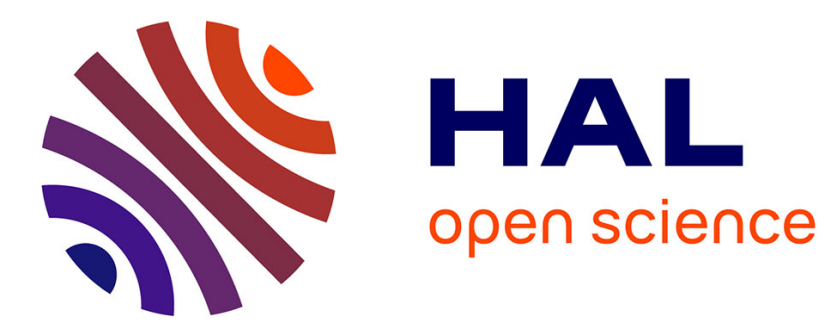

\title{
Comparison of Geometrical Shock Dynamics and Kinematic models for shock wave propagation
}

Julien Ridoux, Nicolas Lardjane, Laurent Monasse, François Coulouvrat

\section{To cite this version:}

Julien Ridoux, Nicolas Lardjane, Laurent Monasse, François Coulouvrat. Comparison of Geometrical Shock Dynamics and Kinematic models for shock wave propagation. Shock Waves, 2017, 28 (2), pp.401-416. 10.1007/s00193-017-0748-2 . hal-01511489

\author{
HAL Id: hal-01511489 \\ https://hal.science/hal-01511489
}

Submitted on 21 Apr 2017

HAL is a multi-disciplinary open access archive for the deposit and dissemination of scientific research documents, whether they are published or not. The documents may come from teaching and research institutions in France or abroad, or from public or private research centers.
L'archive ouverte pluridisciplinaire HAL, est destinée au dépôt et à la diffusion de documents scientifiques de niveau recherche, publiés ou non, émanant des établissements d'enseignement et de recherche français ou étrangers, des laboratoires publics ou privés. 


\title{
Comparison of Geometrical Shock Dynamics and Kinematic models for shock wave propagation
}

April 21, 2017

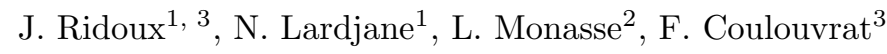 \\ ${ }^{1}$ CEA, DAM, DIF, Arpajon F-91297, France. \\ email: nicolas.lardjane@cea.fr \\ ${ }^{2}$ Université Paris-Est, CERMICS (ENPC),
}

6 et 8 avenue Blaise Pascal, Cité Descartes, Champs sur Marne, 77455, Marne la Vallée, Cedex 2, France.

${ }^{3}$ Sorbonne Universités, Université Pierre et Marie Curie, Paris 06, CNRS, Institut Jean Le Rond d'Alembert, UMR 7190, Paris, France.

\begin{abstract}
Geometrical Shock Dynamics (GSD) is a simplified model for nonlinear shock wave propagation. It is based on the decomposition of the shock front into elementary ray tubes with a simple relation linking its local curvature and velocity. This relation is obtained under the assumption of strong shock in order to neglect the effect of the post-shock flow on the front. More recently, a new simplified model, referenced as the Kinematic model, was proposed. This model is obtained by combining the threedimensional Euler equations and the Rankine-Hugoniot relations at the front, which leads to an equation for the normal variation of the shock Mach number at the wave front. In the same way as GSD, the Kinematic model is closed by neglecting the post-shock flow effects. Although each model's approach is different, we prove here their structural equivalence: the Kinematic model can be rewritten under the form of GSD with a specific $A-M$ relation. Both models are thus compared through a wide variety of examples including experimental data or Eulerian simulations results when available. Attention is drawn to the simple cases of compression ramps and convex corners' diffraction. The analysis is completed by the more complex cases of the diffraction over a cylinder, a sphere, a mound and a trough.
\end{abstract}

Keywords: Shock wave, Geometrical Shock Dynamics, Kinematic model 


\section{Introduction}

The problem of determining the motion of shock waves has received considerable attention since several decades. The development of simplified models, able to estimate the position, shape, and strength of a shock, is of prime importance in several domains such as the pyrotechnics industry, explosion hazards, or noise annoyance among others. In 1957, Whitham published a hyperbolic model called Geometrical Shock Dynamics (GSD) [31, 33, 36] able to estimate correctly the propagation of a strong shock, and working surprisingly well for weak ones in some cases, such as the compression ramp for instance. This model splits the shock front into elementary parts propagating independently along ray tubes. A relation between the local geometry of the shock and its local velocity closes the system. A ray tube is actually assimilated to a channel of cross sectional area $A$ with rigid walls. This allows the determination of a relation between $A$ and the shock Mach number $M$ ( $A-M$ relation), by neglecting the post-shock flow under the strong shock hypothesis. This relation was independently derivated by Chester [7], Chisnell [9] and Whitham [32], and is sometimes called $C C W$ relation. The GSD model was first expressed for a uniform gas at rest $[31,33]$. It has then been extended to the propagation through moving gases [34], and nonuniform flow fields [8,6]. A complete synthesis of this model can be found in [38]. From a numerical point of view, many algorithms have been developed. They are based on front-tracking methods [13, 21], Eulerian conservative schemes [22, 24], or a fast-marching like approach [18].

It is well known that GSD is quite accurate for sustained shock propagation problems. It has been investigated by numerous authors in the past, mainly in case of converging flows [8,10, 6, 13, 23], but also for outdoor propagation [4], and has proven its ability to estimate correctly the leading shock front. However, the GSD model is limited by three assumptions. The first one is the strong shock hypothesis, which turns out to be valid only for Mach numbers larger than 2 . The second one is the decomposition into ray tubes with rigid walls, which implicitly leads to neglect transversal flow along the shock front. The last one is the omission of post-shock flow influence on the shock motion.

Another, more recent simplified model for shock propagation is called the Kinematic model $[25,26,20]$. It is rather based on a mathematical viewpoint. Considering the shock front as a singular surface [29], it is possible to combine the Euler equations and Rankine-Hugoniot relations in order to obtain an evolution equation for the shock intensity. The model naturally takes into account transverse flow along the shock front, and no assumption on the shock strength is necessary. These are desirable properties for a more general model than GSD. This new formalism was first expressed by Wright [37] to give an intrinsic description of unsteady shock waves, and has then been extended to more complex configurations $[26,20]$. However, these studies are limited to theoretical considerations. Only a few numerical cases have been carried out in $1 \mathrm{D}$ radial configurations [25, 1, 27], and 2D for weak shocks [2]. The GSD and Kinematic models share a similar point of view for the shock motion: only the front surface is evolved, which reduces the dimension of the problem and 
leads to fast results. Surprisingly, despite this similarity, no detailed comparison between these two models has been carried out until now to our knowledge.

The main objective of this paper is to compare the Kinematic and GSD models. For this purpose, the derivation of these models is first reviewed in section 2. We then prove their structural similarity in section 3. As properties and analytical solutions for GSD are well known [36], it is natural to transpose them to the Kinematic model to compare the two models. Following [13], a two-dimensional (2D) conservative Lagrangian scheme is developed. It allows numerous comparisons for more complex problems. In section 4, results are systematically compared to experimental data or Eulerian simulation results.

\section{Review of simplified models for shock propa- gation}

The derivation of GSD and Kinematic models are first recalled for the sake of completeness.

\subsection{Problem setup}

We consider the propagation of a three-dimensional shock wave in a uniform, quiescent and calorically perfect gas. The conservation laws for primitive variables read

$$
\begin{cases}\frac{\partial \rho}{\partial t}+\boldsymbol{\nabla} \cdot(\rho \boldsymbol{v}) & =0 \\ \frac{\partial \boldsymbol{v}}{\partial t}+(\boldsymbol{v} \cdot \boldsymbol{\nabla}) \boldsymbol{v}+\rho^{-1} \boldsymbol{\nabla} p= & 0 \\ \frac{\partial p}{\partial t}+\boldsymbol{v} \cdot \boldsymbol{\nabla} p+\rho c^{2} \boldsymbol{\nabla} \cdot \boldsymbol{v} & =0\end{cases}
$$

where $\rho, p$ and $\boldsymbol{v}$ are the density, pressure, and fluid velocity respectively. For a perfect gas, the sound speed $c$ is

$$
c^{2}=\frac{\gamma p}{\rho},
$$

with $\gamma$ the specific heat ratio. These variables are indexed by 0 for the initial state of the gas at rest.

At time $t$, the shock front is identified as the zero level set of a scalar function $\Phi:$

$$
\Gamma(t)=\left\{\boldsymbol{x} \in \Omega \subset \mathbb{R}^{3} / \Phi(\boldsymbol{x}, t)=0\right\} .
$$

In the neighbourhood of the shock, we suppose that $\Phi$ is differentiable and that $\nabla \Phi$ is not identically null. From (3), the unit normal vector at the shock front is defined by

$$
\boldsymbol{n}=\frac{\nabla \Phi}{|\nabla \Phi|},
$$


and $\Phi$ verifies the equation:

$$
\frac{\partial \Phi}{\partial t}+U \boldsymbol{n} \cdot \nabla \Phi=0
$$

where $U$ is the normal speed of the shock. Injecting (4) into (5), we get:

$$
U=-\frac{\partial_{t} \Phi}{|\nabla \Phi|}
$$

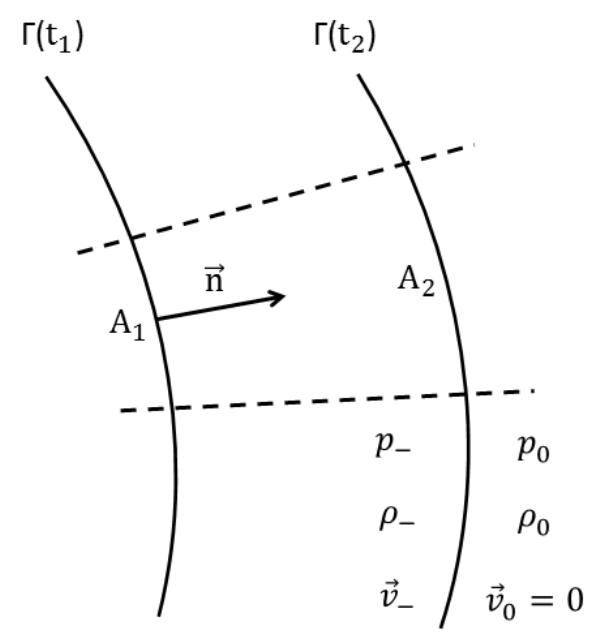

Figure 1: Shock position at times $t_{1}$ and $t_{2}$. Quantities indexed by 0 are fluid parameters of the initial state of the gas, and quantities indexed by - are fluid parameters disturbed by the shock.

Across the shock wave, the jump of a physical quantity $\psi$ is denoted by $[\psi]=\psi_{-}-\psi_{0}$, where $\psi_{-}$is the value of $\psi$ just behind the shock surface. The Rankine-Hugoniot relations [11] link the admissible states of the fluid across the shock. In the particular case considered here, they take the following form [36]:

$$
\begin{aligned}
& \frac{[\rho]}{\rho_{0}}=\frac{2\left(M^{2}-1\right)}{(\gamma-1) M^{2}+2} \\
& \frac{[\boldsymbol{v}]}{c_{0}}=\frac{2\left(M^{2}-1\right)}{(\gamma+1) M} \boldsymbol{n} \\
& \frac{[p]}{p_{0}}=\frac{2 \gamma\left(M^{2}-1\right)}{\gamma+1}
\end{aligned}
$$

with $M$ the shock Mach number: $M=\frac{U}{c_{0}} \geq 1$. 


\subsection{Geometrical Shock Dynamics model}

In Geometrical Shock Dynamics, as sketched in Fig. 1, the shock propagates along rays normal to the front with a local velocity given by the Mach number $M$. The model only addresses the leading shock front, and neglects the influence of post-shock flow.

Assuming a single-pass front, the level set function $\Phi$ is defined as

$$
\Phi(\boldsymbol{x}, t)=\alpha(\boldsymbol{x})-c_{0} t,
$$

where $\alpha$ describes the shock position. The unit normal vector at the shock front, (4), is then

$$
\vec{n}=\frac{\nabla \alpha}{|\nabla \alpha|},
$$

and the normal velocity of the shock, (6), leads to the local eikonal equation

$$
M|\nabla \alpha|=1 .
$$

The GSD model consists in splitting the shock front into elementary areas $A$ propagating along ray tubes in which the cross-flow is neglected. The flow in a ray tube can be expressed mathematically by the equation [33]

$$
\operatorname{div}\left(\frac{\boldsymbol{n}}{A}\right)=0 .
$$

Averaging the equations of the inviscid compressible flow across the tube, one obtains the 1D Euler system with varying cross-section:

$$
\begin{cases}\partial_{t} \rho+v \partial_{r} \rho+\rho \partial_{r} v+\rho v \frac{A^{\prime}(r)}{A(r)} & =0 \\ \partial_{t} v+v \partial_{r} v+\rho^{-1} \partial_{r} p & =0 \\ \partial_{t} p+v \partial_{r} p-c^{2}\left(\partial_{t} \rho+v \partial_{r} \rho\right) & =0\end{cases}
$$

where $r$ is the longitudinal space variable along the tube, $\partial_{t}$ and $\partial_{r}$ denote the partial time and space derivatives respectively, and $A^{\prime}(r)$ stands for the variations of the cross sectional area of the tube. System (12) is rewritten in the following characteristic form :

$$
\begin{aligned}
& \frac{\mathrm{d} p}{\mathrm{~d} t}+\rho c \frac{\mathrm{d} v}{\mathrm{~d} t}+\rho c^{2} v \frac{A^{\prime}(r)}{A}=0 \quad \text { on } C^{+} \quad: \quad \frac{\mathrm{d} r(t)}{\mathrm{d} t}=v+c \\
& \frac{\mathrm{d} p}{\mathrm{~d} t}-\rho c \frac{\mathrm{d} v}{\mathrm{~d} t}+\rho c^{2} v \frac{A^{\prime}(r)}{A}=0 \quad \text { on } C^{-} \quad: \quad \frac{\mathrm{d} r(t)}{\mathrm{d} t}=v-c \\
& \frac{\mathrm{d} p}{\mathrm{~d} t}-c^{2} \frac{\mathrm{d} \rho}{\mathrm{d} t} \quad=0 \quad \text { on } S \quad: \quad \frac{\mathrm{d} r(t)}{\mathrm{d} t}=v .
\end{aligned}
$$


By riding the $C^{+}$characteristic at the shock and using the Rankine-Hugoniot relations (7), the law between $A$ and $M$, called $A-M$ relation, is [5]

$$
\frac{\mathrm{d}_{r} A}{A}+\frac{M \lambda_{W}(M)}{M^{2}-1} \mathrm{~d}_{r} M+f_{W}(M) Q=0,
$$

where $\mathrm{d}_{r}=\frac{\mathrm{d}}{\mathrm{d} r}$ is the derivative along the tube,

$$
\lambda_{W}(M)=\left(1+\frac{2}{\gamma+1} \frac{1-\mu^{2}}{\mu}\right)\left(1+2 \mu+\frac{1}{M^{2}}\right)
$$

and

$$
f_{W}(M)=\frac{\gamma+1}{2} \frac{\mu(\mu-1)}{M\left(M^{2}-1\right)} .
$$

The post-shock Mach number is :

$$
\mu=\sqrt{\frac{(\gamma-1) M^{2}+2}{2 \gamma M^{2}+1-\gamma}},
$$

while $Q=\frac{\left(\partial_{t} p+\rho c \partial_{t} u\right)_{-}}{p_{0} c_{0}^{3}}$ gathers the post-shock flow. $Q$ is an unknown of the problem. As $f_{W}(M)$ tends to 0 when $M$ tends to $+\infty$, Whitham chose to neglect this term under the strong shock assumption. Doing so, the difficult calculation of the post-shock flow is avoided, and a simple relation links $A$ to $M$ :

$$
\frac{\mathrm{d}_{r} A}{A}+\frac{M \lambda_{W}(M)}{M^{2}-1} \mathrm{~d}_{r} M=0
$$

Equation (18) is verified in each ray tube and closes the system (9)-(11). This system defines the geometrical structure of the shock front, and the $A-M$ relation reflects its local dynamics. Some other $A-M$ closures have been studied in the past, like in [30] for noise transmission along shock-waves, in [19] where viscosity is taken into account, or in [5] where the author introduces a post-shock flow model. Here, we restrict ourselves to the classical $A-M$ formulation.

In $2 \mathrm{D}$, the model can be rewritten in the local basis belonging to the shock:

$$
\left\{\begin{array}{l}
\frac{\partial \theta}{\partial \beta}-\frac{A^{\prime}(M)}{M} \frac{\partial M}{\partial \alpha}=0 \\
\frac{\partial \theta}{\partial \alpha}+\frac{1}{A(M)} \frac{\partial M}{\partial \beta}=0
\end{array}\right.
$$

where $\alpha$ and $\beta$ denote the shock position and the ray position respectively. $\theta$ is the angle between rays and $(O x)$ axis (see Fig. 2). At the shock front, $\alpha=c_{0} t$ in coherence with (8), and since $A$ measures the distance between rays, $A \mathrm{~d} \beta$ is actually the curvilinear abscissa along the shock. Using (19), it is easy to prove 


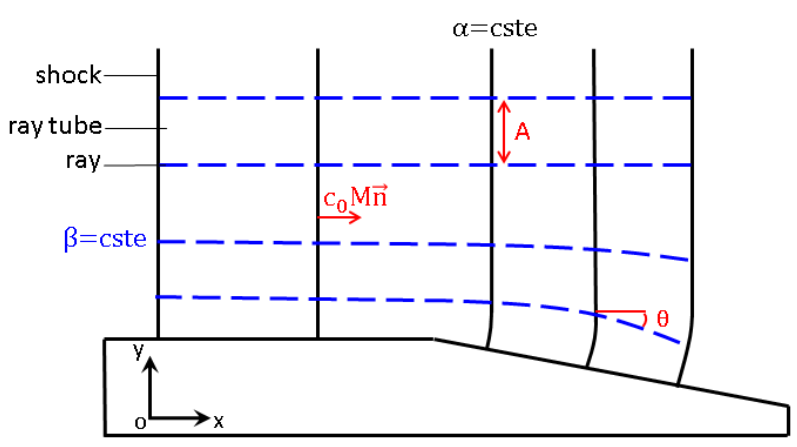

Figure 2: 2D shock wave propagation in Geometrical Shock Dynamics theory. Black lines are successive shock positions and correspond to $\alpha$ coordinates. Blue dashed lines are rays and correspond to $\beta$ coordinates. $\theta$ is the angle between rays and (Ox) axis. Rays delimit ray tubes of area $A$.

that the GSD model is hyperbolic provided that $A^{\prime}(M)<0$. Discontinuities can thus develop on the shock front. They correspond to the triple point position when a Mach stem arises, and are called shock-shocks by Whitham. A detailed analysis of the Riemann problem for (19) can be found in [18].

\subsection{Kinematic model}

Another simplified model for shock front propagation is the Kinematic (KIN) model [26]. It relies on a more mathematical vision of the shock motion, using the singular surface theory [29]. As GSD, it describes only the leading shock front. This model is based on a combination of the 3D Euler equations (1) applied in the vicinity of the shock and the Rankine-Hugoniot relations (7) at the front. This allows to take into account the cross-flow at the shock, and no assumption on the shock strength is necessary, which is a desirable property.

The Kinematic model was initially expressed by Wright in 1974 for a perfect gas [37], and has been reformulated and detailed by Sharma in 1994 [26]. It has then been extended to van der Waals equation of state in 2009 [20]. Only a few studies for one-dimensional planar and non-planar shock waves can be found in $[25,1,27]$, and for 2D weak shocks in [2].

Here, the shock is considered as a singular surface (3) moving with its normal velocity $U \boldsymbol{n}$. The shock position, $\boldsymbol{x}_{\mathrm{sh}}$, is then defined by

$$
\frac{\mathrm{d} \boldsymbol{x}_{\mathrm{sh}}}{\mathrm{d} t}=c_{0} M \boldsymbol{n},
$$

which is equivalent to (10) taking $\alpha\left(\boldsymbol{x}_{\mathrm{sh}}\right)=c_{0}$ ( (see eq. (8)). Let $\psi$ be a physical quantity, $\rho$ or $\boldsymbol{v}$ for instance. We define the temporal rate of change of $\psi$ following the shock as:

$$
\frac{\mathrm{d} \psi}{\mathrm{d} t}=\frac{\partial \psi}{\partial t}+U(\boldsymbol{n} \cdot \nabla \psi)
$$


and its spatial rate of change along the shock surface:

$$
\boldsymbol{\nabla}^{S} \psi=\nabla \psi-\boldsymbol{n}(\boldsymbol{n} \cdot \nabla \psi) .
$$

If $\boldsymbol{\psi}$ is a vector, we can also define the divergence operator along the shock surface:

$$
\boldsymbol{\nabla}^{S} \cdot \boldsymbol{\psi}=\boldsymbol{\nabla} \cdot \boldsymbol{\psi}-\boldsymbol{n} \cdot(\boldsymbol{n} \cdot \boldsymbol{\nabla} \boldsymbol{\psi})
$$

By definition of (22), we have

$$
\boldsymbol{n} \cdot \nabla^{S} \psi=0 .
$$

As $\boldsymbol{n}$ is a unit vector, $\boldsymbol{n} \cdot \boldsymbol{n}=1$, one gets for any differential operator $\mathcal{D}$, such as $\partial_{t}$ or $\boldsymbol{n} \cdot \boldsymbol{\nabla}$ for instance:

$$
\boldsymbol{n} \cdot \mathcal{D} \boldsymbol{n}=0 .
$$

From (23) and (25), one finds:

$$
\boldsymbol{\nabla}^{S} \cdot \boldsymbol{n}=\boldsymbol{\nabla} \cdot \boldsymbol{n} .
$$

This last relation combined with (24) gives:

$$
\boldsymbol{\nabla}^{S} \cdot(f \boldsymbol{n})=f \boldsymbol{\nabla} \cdot \boldsymbol{n},
$$

where $f$ is any scalar function.

From the singular surface theory [29], (21)-(23) give the following compatibility conditions holding on the shock front:

$$
\begin{gathered}
\frac{\mathrm{d}[\psi]}{\mathrm{d} t}=\left[\frac{\partial \psi}{\partial t}\right]+U[\boldsymbol{n} \cdot \boldsymbol{\nabla} \psi] \\
\boldsymbol{\nabla}^{S}[\psi]=[\boldsymbol{\nabla} \psi]-\boldsymbol{n}[\boldsymbol{n} \cdot \boldsymbol{\nabla} \psi]
\end{gathered}
$$

and if $\boldsymbol{\psi}$ is a vector:

$$
\boldsymbol{\nabla}^{S} \cdot[\boldsymbol{\psi}]=[\boldsymbol{\nabla} \cdot \boldsymbol{\psi}]-\boldsymbol{n} \cdot[\boldsymbol{n} \cdot \boldsymbol{\nabla} \boldsymbol{\psi}] .
$$

These relations allow to track the jump relation of the physical quantity $\psi$ along the shock front during its propagation. Furthermore, it can easily be shown that for two quantities $f$ and $g$ :

$$
[f g]=[f] g_{-}+f_{+}[g] .
$$

Applying the Euler equations (1) at the shock, and using relations (28)-(31), one finds after lengthy but straightforward calculations,

$$
\begin{aligned}
\frac{\mathrm{d}[\boldsymbol{v}]}{\mathrm{d} t}+([\boldsymbol{v}] \cdot \boldsymbol{n}-U)[\boldsymbol{n} \cdot \boldsymbol{\nabla} \boldsymbol{v}] & \\
& +\frac{1}{\rho_{-}}\left(\boldsymbol{\nabla}^{S}[p]+\boldsymbol{n}[\boldsymbol{n} \cdot \boldsymbol{\nabla} p]\right)=0
\end{aligned}
$$


and

$$
\begin{aligned}
\frac{\mathrm{d}[p]}{\mathrm{d} t}+([\boldsymbol{v}] \cdot \boldsymbol{n}-U)[\boldsymbol{n} \cdot \boldsymbol{\nabla} p] & \\
& +\gamma p_{-}\left(\boldsymbol{\nabla}^{S} \cdot[\boldsymbol{v}]+\boldsymbol{n} \cdot[\boldsymbol{n} \cdot \boldsymbol{\nabla} \boldsymbol{v}]\right)=0 .
\end{aligned}
$$

The derivation of the Rankine-Hugoniot relations (7) following the shock motion yields:

$$
\begin{aligned}
& \frac{1}{c_{0}} \frac{\mathrm{d}[\boldsymbol{v}]}{\mathrm{d} t}=\frac{2}{\gamma+1} \frac{\left(M^{2}+1\right)}{M} \frac{\mathrm{d} M}{\mathrm{~d} t} \boldsymbol{n}+\frac{2}{\gamma+1} \frac{\left(M^{2}-1\right)}{M} \frac{\mathrm{d} \boldsymbol{n}}{\mathrm{d} t} \\
& \frac{1}{p_{0}} \frac{\mathrm{d}[p]}{\mathrm{d} t}=\frac{4 \gamma}{\gamma+1} M \frac{\mathrm{d} M}{\mathrm{~d} t} .
\end{aligned}
$$

The term $\boldsymbol{n} \cdot[\boldsymbol{n} \cdot \boldsymbol{\nabla} \boldsymbol{v}]$ in (33) is eliminated through the combination $([\boldsymbol{v}] \cdot \boldsymbol{n}-$ $U)(33)-\gamma p_{-} \boldsymbol{n} \cdot(32)$, which yields to

$$
\begin{aligned}
&([\boldsymbol{v}] \cdot \boldsymbol{n}-U) \frac{\mathrm{d}[p]}{\mathrm{d} t}-\gamma p_{-} \boldsymbol{n} \cdot \frac{\mathrm{d}[\boldsymbol{v}]}{\mathrm{d} t} \\
&+\left\{([\boldsymbol{v}] \cdot \boldsymbol{n}-U)^{2}\right. \\
&\left.-\frac{\gamma p_{-}}{\rho_{-}}\right\}[\boldsymbol{n} \cdot \boldsymbol{\nabla} p] \\
&+\gamma p_{-}([\boldsymbol{v}] \cdot \boldsymbol{n}-U) \boldsymbol{\nabla}^{S} \cdot[\boldsymbol{v}]=0 .
\end{aligned}
$$

Finally, injecting (34) and (7) into (35), and using properties (24)-(27), the equation for the Mach number variation is obtained:

$$
\frac{\mathrm{d} M}{\mathrm{~d} t}=-c_{0} \frac{M^{2}-1}{\lambda_{K}(M)}\left(\operatorname{div}(\boldsymbol{n})+f_{K}(M) \frac{\left(\partial_{n} p\right)_{-}}{p_{0}}\right),
$$

where $\operatorname{div}(\boldsymbol{n})$ is the local curvature of the front and

$$
f_{K}(M)=\frac{(\gamma+1)^{2}}{2 \gamma\left(2 \gamma M^{2}+1-\gamma\right)} .
$$

The term $\left(\partial_{n} p\right)_{-}=(\boldsymbol{n} \cdot \boldsymbol{\nabla} p)_{-}$gathers the post-shock flow effect. For the Kinematic model, the $\lambda$ function reads

$$
\lambda_{K}(M)=\frac{(\gamma+1)\left\{2(2 \gamma-1) M^{4}+(\gamma+5) M^{2}+1-\gamma\right\}}{\left(2 \gamma M^{2}+1-\gamma\right)\left(2+(\gamma-1) M^{2}\right)} .
$$

The normal at the shock, $\boldsymbol{n}$, is also an unknown of the problem. Differentiating $|\nabla \Phi|=\sqrt{\Phi_{, k} \Phi_{, k}}$ with respect to $\mathcal{D}$, we deduce

$$
\mathcal{D}|\vec{\nabla} \Phi|=\vec{n} \cdot \vec{\nabla}(\mathcal{D} \Phi) .
$$


Differentiating (4) following the shock motion (21), and using relations (39) (22), leads after some calculations to

$$
\frac{\mathrm{d} \boldsymbol{n}}{\mathrm{d} t}=-\nabla U+\boldsymbol{n}(\boldsymbol{n} \cdot \boldsymbol{\nabla} U)=-c_{0} \boldsymbol{\nabla}^{S} M,
$$

Under the hypothesis that the post-shock flow influence over the shock $(\boldsymbol{n} \cdot \boldsymbol{\nabla} p)_{-}$is known, the Kinematic model is thus composed of equations (20), (36) and (40).

\section{Properties and integration of the GSD and KIN models}

The Kinematic model can be rewritten in a similar form to GSD, which allows an easy comparison between the models.

\subsection{Structural equivalence of the GSD and KIN models}

Noticing that $M=M\left(\boldsymbol{x}_{\mathrm{sh}}\right)$, and using (20), we have

$$
\frac{\mathrm{d} M}{\mathrm{~d} t}=\left.\frac{\mathrm{d} M}{\mathrm{~d} t}\right|_{\boldsymbol{x}_{\mathrm{sh}}(t)}=\frac{\mathrm{d} \boldsymbol{x}_{\mathrm{sh}}(t)}{\mathrm{d} t} \cdot \nabla M=c_{0} M \boldsymbol{n} \cdot \nabla M,
$$

As $M$ is a quantity defined only at the shock, $\boldsymbol{n} \cdot \boldsymbol{\nabla}$ can be regarded as the spatial derivative along the normal direction following the shock. We choose to note $\frac{\mathrm{d}}{\mathrm{d} n}$ this derivative, which corresponds to $\frac{\mathrm{d}}{\mathrm{d} r}$ in the $1 \mathrm{D}$ case.

It is easy to verify that (36) reduces to (11) with a similar A-M relation (14). Indeed, let us define two nonzero functions, $h$ and $H$, such that $h \times(36)$ can be written as $\operatorname{div}(H \boldsymbol{n})=0$. We find by identification:

$$
H=\frac{M^{2}-1}{M \lambda_{K}(M)} h
$$

and

$$
\boldsymbol{n} \cdot \boldsymbol{\nabla} H=h\left(\boldsymbol{n} \cdot \boldsymbol{\nabla} M+\frac{M^{2}-1}{M \lambda_{K}(M)} f_{K}(M) \frac{\left(\partial_{n} p\right)_{-}}{p_{0}}\right) .
$$

Dividing (43) by (42) and with notation $A=\frac{1}{H}$, it yields to

$$
\frac{1}{A} \frac{\mathrm{d} A}{\mathrm{~d} n}+\frac{M \lambda_{K}(M)}{M^{2}-1} \frac{\mathrm{d} M}{\mathrm{~d} n}+f_{K}(M) Z=0,
$$

with $Z=\frac{\left(\partial_{n} p\right)_{-}}{p_{0}}$. We are thus able to decompose the shock front into elementary ray tubes of cross sectional area $A$ for the KIN model, with a specific $A-M$ relation. The term $Z$ takes into account the influence of post shock flow over the shock front. The function $f_{K}(M)$ is defined for $M \geq 1$ and is decreasing from $\frac{\gamma+1}{2 \gamma}$ for $M=1$, to 0 for infinite Mach number. If we assume that post-shock 
influence is small (step shock hypothesis), $f_{K}(M) Z$ can be neglected in (44). Doing so, we avoid the difficult calculation of post shock flow, in the same way as Whitham's theory, and we can easily compare both models.

Finally, the GSD and KIN models can be written using the eikonal equation (10), which gives the shock position, the relation (11) defining the ray tube and an $A-M$ relation describing the local variations of the shock front. The latter is given by:

$$
\frac{\mathrm{d} A}{A}+\frac{M \lambda(M)}{M^{2}-1} \mathrm{~d} M=0,
$$

with $\lambda(M)=\lambda_{W}(M)$ for GSD, and $\lambda(M)=\lambda_{K}(M)$ for the KIN model. The structural equivalence of the KIN model with GSD allows a very easy comparison, through analytical solutions [36] and numerical schemes [13] initially developed for GSD.

\subsection{Main properties}

$A-M$ relation. The difference between the GSD and KIN models fundamentally comes from the way the $A-M$ relation is defined. Whitham's approach consists in riding the $C^{+}$characteristic at the shock, while the Kinematic approach is based on a combination of these characteristics, using all available flow information. It results that the post-shock effects terms, namely $Q$ for GSD and $Z$ for the KIN model, are different. Consequently, these models do not neglect the same information. This point was already pointed out in [5].

Under the hypothesis $Q=0$ and $Z=0$, the shock motion is only driven by the functions $\lambda_{W}$ or $\lambda_{K}$, as drawn in Fig. 3. In the weak shock limit $(M \approx 1)$, we have:

$$
\lambda_{W} \sim \lambda_{K} \sim 4 .
$$

For $M<2$, the values of $\lambda_{W}$ and $\lambda_{K}$ are close, so both models are nearly equivalent in this regime. For $M>2$,

$$
\lambda_{K}(M) \geq \lambda_{W}(M),
$$

and the strong shock limits $(M>>1)$ are different. For $\gamma=1.4$, they are:

$$
\begin{aligned}
& \lambda_{W} \sim 1+\frac{2}{\gamma}+\sqrt{\frac{2 \gamma}{\gamma-1}} \approx 5.0743 \\
& \lambda_{K} \sim \frac{(\gamma+1)(2 \gamma-1)}{\gamma(\gamma-1)} \approx 7.7143 .
\end{aligned}
$$

This is the main difference between the two approaches. The GSD model rapidly reaches the strong shock regime, which is coherent with the underlying hypothesis of the model. The KIN model has a larger transitory phase, and reaches its strong shock limit for much higher Mach numbers. Therefore, it should lead to distinct solutions at intermediate and high Mach numbers. 


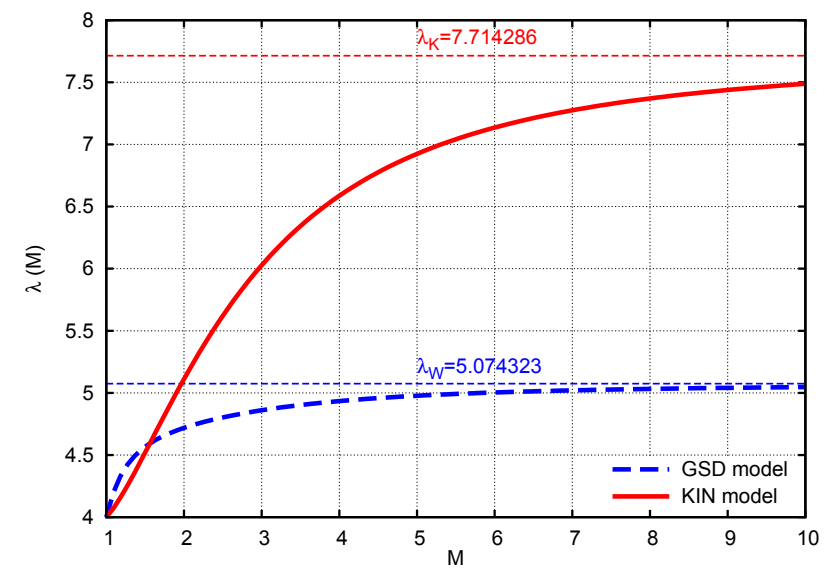

Figure 3: Representation of $\lambda(M)$ for the GSD and KIN models.

From (45), we can consider $A$ as dimensionless. A direct integration gives

$$
A(M)=\frac{g(M)}{g\left(M_{0}\right)},
$$

with $M_{0}$ the initial Mach number, and

$$
g(M)=\exp \left(-\int \frac{M \lambda(M)}{M^{2}-1} \mathrm{~d} M\right) .
$$

There is no simple analytical expression of (48) for GSD, but for KIN we have:

$$
g_{\mathrm{KIN}}(M)=\frac{\left(2 \gamma M^{2}+1-\gamma\right)^{\frac{\gamma-1}{2 \gamma}}}{\left(M^{2}-1\right)^{2}\left(2+(\gamma-1) M^{2}\right)^{\frac{3-\gamma}{2(\gamma-1)}}} .
$$

The variation of the cross sectional area $A$, with respect to Mach number $M$, is plotted in Fig. 4 for $M_{0}=1.1$. As expected, one can notice a similar behaviour of the models for $M \leq 2$, due to close $\lambda$ values. From $M \approx 2, \mathrm{KIN}$ cross sectional area decreases more rapidly than in the case of GSD, due to property (46). Models should consequently give similar solutions for $M \leq 2$, and distinct solutions for Mach numbers larger than 2 .

Hyperbolic nature. It is well known that GSD is a hyperbolic system provided that $A^{\prime}(M)<0$ [36]. This property is verified from (45), as $\lambda(M) \geq 4$ and $M \geq 1$. Consequently, the KIN model is also hyperbolic. It means that waves may develop on the shock front which are responsible for the modification of its intensity, shape and orientation. In particular shock-shocks can develop as in the GSD model. 


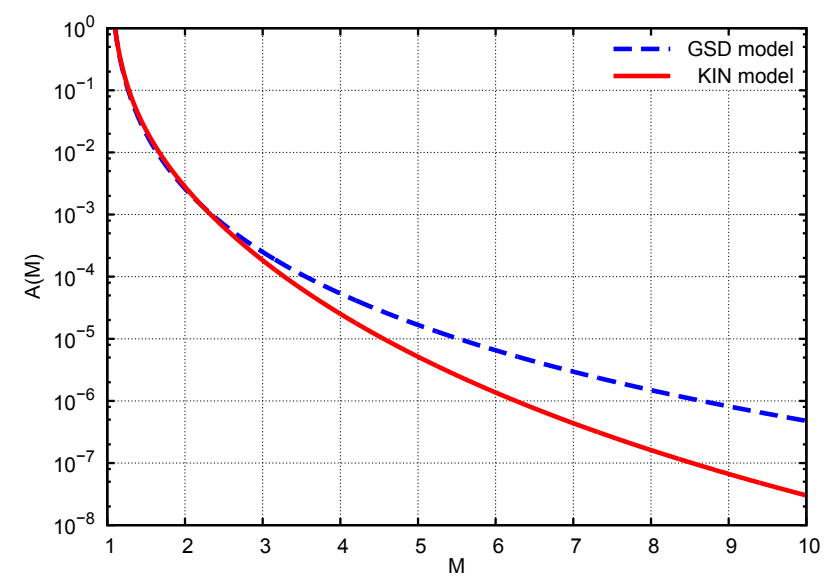

Figure 4: $A(M)$ for the GSD and KIN models with an initial Mach number $M_{0}=1.1$ and $\gamma=1.4$.

2D diffraction. In the 2D case, the models can be written under the form (19). The characteristic form is

$$
\left\{\frac{\partial}{\partial \alpha} \pm u(M) \frac{\partial}{\partial \beta}\right\}(\theta \pm \omega(M))=0,
$$

where

$$
\omega(M)=\int_{1}^{M} \sqrt{\frac{\lambda(m)}{m^{2}-1}} \mathrm{dm}<+\infty .
$$

The perturbations travel along the shock front with the velocity, defined in the shock local basis,

$$
\frac{\mathrm{d} \beta}{\mathrm{d} \alpha}=u(M)=\frac{1}{A(M)} \sqrt{\frac{M^{2}-1}{\lambda(M)}} .
$$

From (50), the Riemann invariants are:

$$
\theta \pm \omega(M)=c s t \quad \text { on } \quad C^{ \pm}: \frac{\mathrm{d} \beta}{\mathrm{d} \alpha}=u(M) .
$$

Let us consider the diffraction of a planar shock at Mach number $M_{0} \geq 1$ over a wall of angle $\theta_{w}$ (see Fig. 5 for a convex wall $\theta_{w}<0$ ). The initial trajectory angle is $\theta_{0}=0$. The problem is analogous to one dimensional nonlinear gas dynamics problems [35]. All $C^{-}$characteristics cross the initial shock and the solution is a simple wave. Along $C^{-}$, we have from equation (53),

$$
\theta-\theta_{0}=\omega(M)-\omega\left(M_{0}\right) .
$$

If the wall is concave $\theta_{w}>0$, then the rays tend to cross, so a compression wave forms on the shock front and a shock-shock potentially appears. From (54), we 


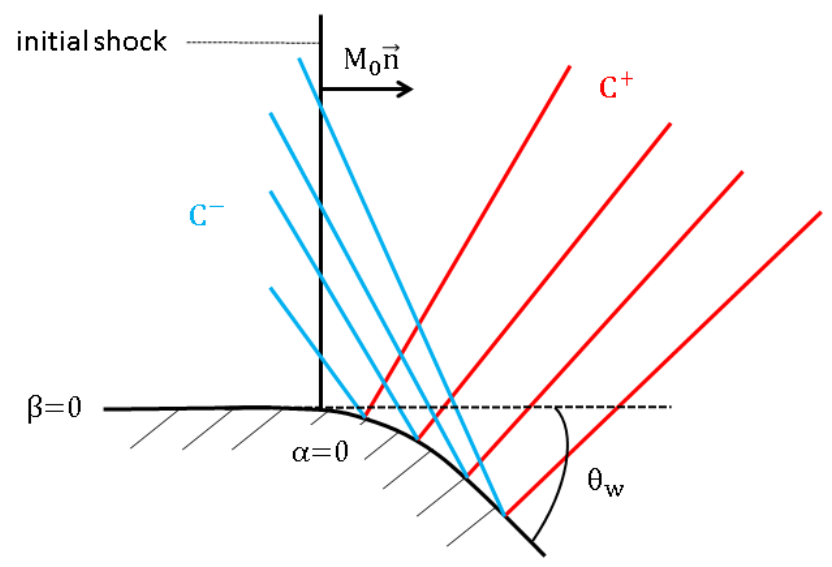

Figure 5: Diffraction of a planar shock at Mach number $M_{0}$ over a convex wall of angle $\theta_{w}<0$. All $C^{-}$characteristics cross the initial shock; the solution is then a simple wave.

have

$$
\omega(M) \geq \omega\left(M_{0}\right),
$$

and as $M \rightarrow \omega(M)$ is an increasing function, the Mach number increases. In particular, we always have a solution such that $M \geq 1$. Conversely, for a convex wall $\theta_{w}<0$, as sketched in Fig. 5, the rays tend to spread out. This expansion wave decreases the Mach number intensity from $M_{0}$ to $M_{w}$ at the wall. In this case, for a given $\theta_{w}$ angle, there is no solution for sufficiently weak shock. Indeed from (54), there exists a solution such that $M \geq 1$ if and only if

$$
M_{0} \geq M_{l i m} \quad \text { with } \quad \int_{1}^{M_{l i m}} \sqrt{\frac{\lambda(m)}{m^{2}-1}} \mathrm{~d} m=-\theta_{w} .
$$

This is the main limitation of the GSD model, and in fact also of the Kinematic model, as can be seen in Fig. 6 . For any given data $\left(M_{0}, \theta_{w}\right)$ in the area below the curves in this figure, there is no solution for the diffraction of a planar shock over a convex wall. These curves are similar for both models.

Waves velocity. The physical velocity of the perturbations (52) is defined by

$$
\frac{A \mathrm{~d} \beta}{\mathrm{d} t}=c_{0} A(M) u(M)=c_{0} \sqrt{\frac{M^{2}-1}{\lambda(M)}} .
$$

We denote by $a_{\mathrm{GSD}}$ and $a_{\mathrm{KIN}}$ this velocity for the GSD $\left(\lambda=\lambda_{W}\right)$ and KIN $\left(\lambda=\lambda_{K}\right)$ models respectively. 


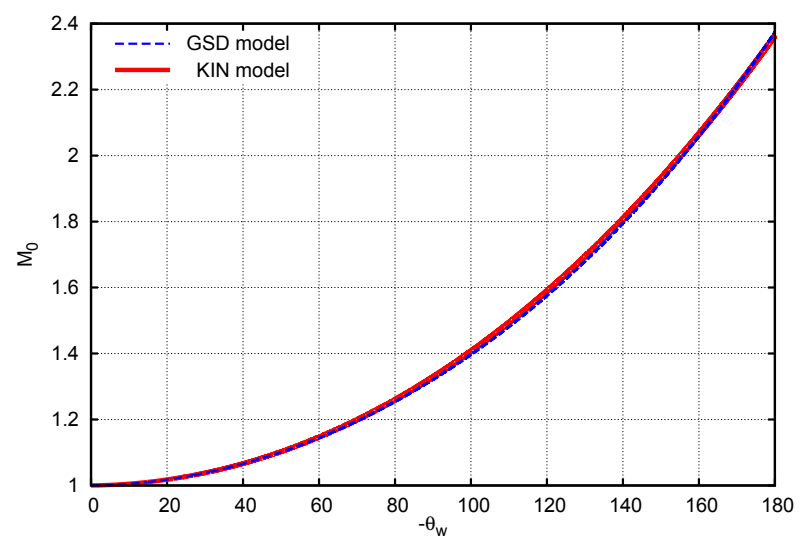

Figure 6: Minimal value for the initial Mach number $M_{0}$ with respect to $\theta_{w}$ in order for a solution to exist for the planar shock diffraction problem over a convex wall (see condition (55)). There is no solution if $\left(M_{0}, \theta_{w}\right)$ is strictly below the curve.

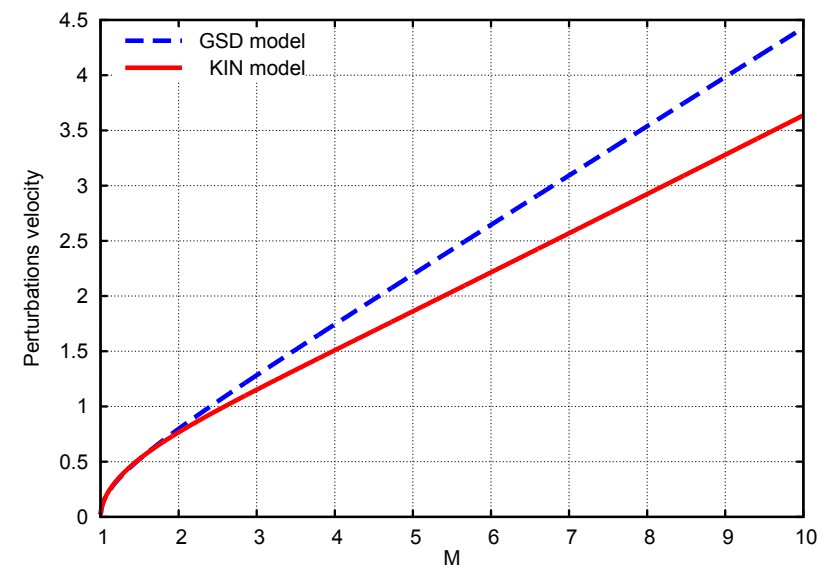

Figure 7: Dimensionless perturbations velocity for the GSD $\left(a_{\mathrm{GSD}} / c_{0}\right)$ and KIN models $\left(a_{\mathrm{KIN}} / c_{0}\right)$. 
The dimensionless perturbation velocities are drawn in Fig. 7. In the weak shock limit $(M \rightarrow 1)$, both models have the same perturbation velocity:

$$
\frac{a_{\mathrm{GSD}}}{c_{0}} \sim \frac{a_{\mathrm{KIN}}}{c_{0}} \sim \sqrt{\frac{M-1}{2}},
$$

These velocities remain very close while $M \leq 2$, and gradually separate then. The biggest difference is observed in the strong shock limit. In this case, the perturbation velocities for both models are:

$$
\frac{a_{\mathrm{GSD}}}{c_{0}} \sim 0.443926 M \quad \text { and } \quad \frac{a_{\mathrm{KIN}}}{c_{0}} \sim 0.360041 M
$$

for $\gamma=1.4$. Thus, the perturbations travel faster for GSD than the KIN model. Consequently, for an expansion wave, the Mach number decrease will be less important for the KIN approach, and in the compression case, shock-shocks will travel slower on the front for KIN.

\subsection{Numerical scheme}

Before going to further comparison of the models, we briefly describe the numerical scheme used to calculate the successive positions of the shock when no analytical solution exists. The following scheme, initially developed for GSD, can also be used for KIN since it can be formulated in a strictly equivalent way.

The numerical scheme is inspired from Henshaw et al. [13]. It is a natural approach based on a Lagrangian vision of the shock and on its decomposition in ray tubes. The scheme is robust, precise and conservative.

In $2 \mathrm{D}$, the shock front at time $t$ is approximated by a finite set of points $\left\{\boldsymbol{x}_{i}(t), i=1, \ldots, N\right\}$. Each point is associated with a ray tube of local area $A_{i}(t)$, a Mach number $M_{i}(t)$, and a normal vector $\boldsymbol{n}_{i}(t)$. The local area is calculated geometrically, and $M_{i}(t)$ is obtained by integrating numerically the $A-M$ relation (45) on each ray from the initial time $t_{0}$ to $t$. An interpolation of the shock surface is performed using a monotone cubic method [14] in order to avoid the creation of new extrema. The unit normal vector, $\left\{\boldsymbol{n}_{i}(t), i=1, \ldots, N\right\}$, is calculated accordingly. Each point is then moved with a fourth-order RungeKutta scheme to time $t+d t$ by considering the discrete version of (20). After this propagation step, points are inserted in expansion regions to maintain acceptable surface resolution, and are deleted in compression regions in order to avoid intersecting rays and fit shock-shocks. Finally, a simple smoothing procedure is applied to dampen the high-frequency errors in $\boldsymbol{x}_{i}(t)$. In the presence of obstacles, wall boundary conditions must be applied in order to keep the shock locally normal to the boundary. This is achieved by clamping the spline representing the shock front at the boundary. The reader is referred to [13] for technical details. 


\section{Results and comparison}

In this section, we present some 2D comparisons between the KIN and GSD models. Results are systematically compared with experimental data and Eulerian simulations results when possible. The latter are provided by the HERA Computational Fluid Dynamics software [15]. HERA is a multi-physics code using adaptive mesh refinement (AMR). It has already been used with success for strong shock interaction with obstacles [12], and weak shock propagation over long distances [17]. During a simulation, the first arrival time of the shock front is recorded in each computational cell, and the leading front at time $t$ is extracted with a marching square algorithm [16]. This allows to estimate geometrically the local shock's Mach number by calculating the instantaneous velocity of the front from time $t$ to $t+\mathrm{d} t$.

\subsection{Semi-analytical solutions}

We first study the diffraction of a planar shock over a corner. This is a simple case analogous to the elementary Riemann problem. For a convex corner, the solution is a simple wave and is continuous, while for a concave corner, a shockshock appears on the shock front. These problems are well detailed in [36] and [18].

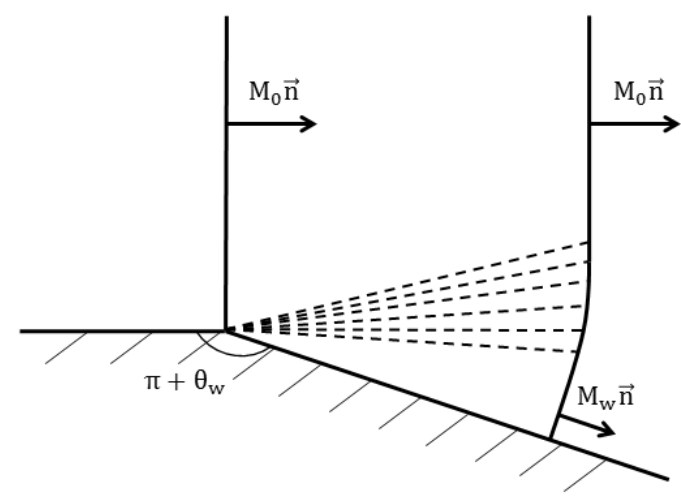

Figure 8: Scheme of a planar shock diffraction over a convex corner of angle $\theta_{w}<0$. An expansion wave develops on the shock front, decreasing the Mach number value from $M_{0}$ to $M_{w}$ at the wall.

Continuous case. Let us consider an initially planar shock travelling at Mach number $M_{0}$ and diffracting over a convex corner of angle $\theta_{w}<0$ (see Fig. 8). The solution is continuous and develops expansion waves, splitting the front in three parts: an undisturbed part, a constant planar part near the wall, and a curved transition zone in between. The solution $(M, \theta)$ of the problem is a 


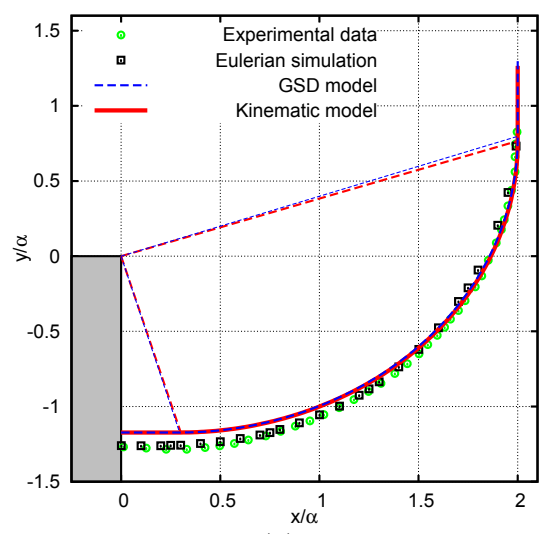

(a)

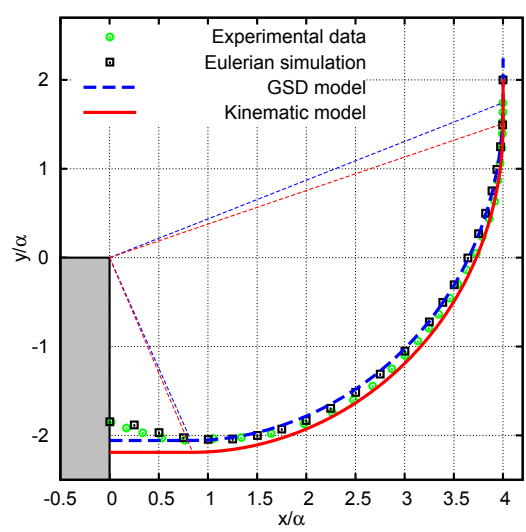

(b)

Figure 9: Diffraction of a planar shock over a convex corner of angle -90 degrees at Mach numbers 2 (a) and 4 (b). The grey rectangle represents the wall. The shock travels from left to right. Comparison of the GSD and KIN models with experimental data from [36] and Eulerian simulations.

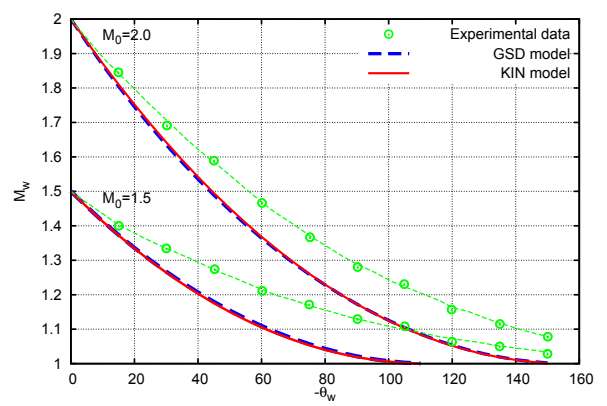

(a)



(b)

Figure 10: Diffraction of planar shock over a convex corner. Wall Mach number with respect to deflection angle $\theta_{w}$, in degree, at Mach numbers 1.5 and 2 (a) and 3 and 4 (b). Experimental data come from [28]. 
simple wave, given by

$$
\mid \begin{array}{lll}
M=M_{0} & , \theta=\theta_{0} & \text { if } u\left(M_{0}\right) \leq \frac{\beta}{\alpha} \\
u(M)=\frac{\beta}{\alpha} & , \theta=\int_{M_{0}}^{M} \frac{d m}{A u} & \text { if } u\left(M_{w}\right) \leq \frac{\beta}{\alpha} \leq u\left(M_{0}\right) \\
\theta_{w}=\int_{M_{0}}^{M_{w}} \frac{d m}{A u} \quad, \theta=\theta_{w} & \text { if } \frac{\beta}{\alpha} \leq u\left(M_{w}\right),
\end{array}
$$

where the function $u$ is defined by (52).

In Fig. 9, we present solutions for an angle $\theta_{w}=-90$ degrees at Mach numbers $M_{0}=2$ (Fig. 9-a) and $M_{0}=4$ (Fig. 9-b). They are obtained by using a Simpson method to approximate the integrals. One can see the good agreement of Whitham and Kinematic theories with the experimental data from [36] and the Eulerian simulation results. As expected, the models are very close for a moderate shock (Fig. 9-a), due the close values of their respective wave velocities. They exhibit differences for higher Mach numbers (Fig. 9-b). The shock is faster with the KIN model due to the fact that perturbations travel slower on the shock front than for the GSD model. This causes the shock intensity to decrease. In this case, the GSD model seems slightly closer to the experimental and numerical results. At higher Mach numbers, one can note the existence of an inflexion point on the front near the wall on experimental and Eulerian results. As detailed in [5], the post-shock effects, not taken into account in GSD or KIN, are responsible for this behaviour.

The wall Mach number $M_{w}$ is plotted in Fig. 10 with respect to the angle of the corner $\theta_{w}$ for different initial Mach numbers $M_{0}$. Results are compared with experimental data from [28]. Again, the GSD and KIN models are similar for weak and moderate shocks (Fig. 10-a). They both underestimate the experimental results. Moreover, one can observe the limitation discussed in section 3.2: once the wall Mach number reaches 1 for a given $\theta_{w}$, no solution such that $M_{w} \geq 1$ exists anymore for smaller $\theta_{w}$ values. For larger $M_{0}$, one can see that the wall Mach number calculated by the KIN approach is higher than the GSD one (Fig. 10-b). This is explained by the fact that waves on the shock travel slower with the KIN model. For both models, the results are in good agreement with experimental data at $M_{0}=3$. At $M_{0}=4$, they overestimate $M_{w}$ and the GSD model is better.

Shock-shock occurence. Let us now consider the diffraction of a planar shock at Mach number $M_{0}$ over a concave corner of deflection angle $\theta_{w}>0$ (see Fig. 11). We note $\chi$ the angle of the shock-shock trajectory with the $(O x)$ axis. 


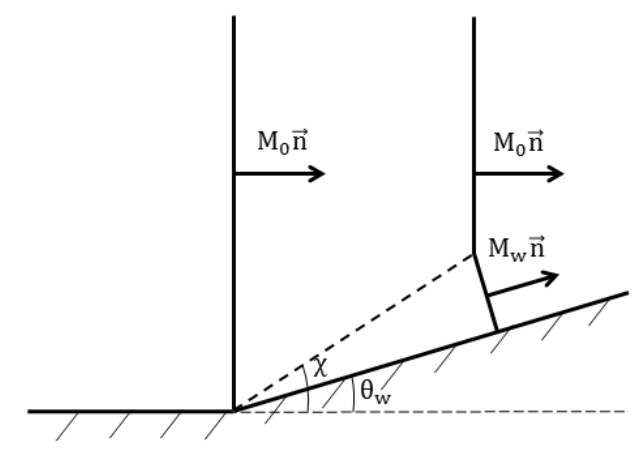

Figure 11: Scheme of a planar shock diffraction over a concave corner of angle $\theta_{w}>0$. A shock-shock appears immediately on the shock front, increasing the shock Mach number from $M_{0}$ to $M_{w}$ at the wall.

The solution $\left(M_{w}, \chi\right)$ of this problem is given implicitly by

$$
\begin{aligned}
\tan \left(\theta_{w}\right) & =\frac{\sqrt{\left(\left(\frac{M_{w}}{M_{0}}\right)^{2}-1\right)\left(1-\left(\frac{A_{w}}{A_{0}}\right)^{2}\right)}}{1+\frac{A_{w} M_{w}}{A_{0} M_{0}}} \\
\tan \left(\chi-\theta_{w}\right) & =\frac{A_{w}}{A_{0}} \sqrt{\frac{1-\left(\frac{M_{0}}{M_{w}}\right)^{2}}{1-\left(\frac{A_{w}}{A_{0}}\right)^{2}}}
\end{aligned}
$$

where $\frac{A_{w}}{A_{0}}=A\left(M_{w}\right)$ is defined by (47).

We have no experimental data reported for this problem, only Eulerian simulation results are considered for comparison. The successive shock positions and the shock-shock trajectory are presented in Fig. 12 for $\theta_{w}=20$ degrees. One can note the excellent agreement of GSD for this example. At Mach number 2 (Fig. 12-a), the shock-shock trajectory is identical for the GSD model and the Eulerian simulation. The KIN model is also very close. At Mach number 4 (Fig. 12-b), the GSD model remains close to the Eulerian simulation results, while the KIN model underestimates the shock-shock position. We draw the wall Mach number $M_{w}$ with respect to the deflection angle $\theta_{w}$ at Mach numbers 2 and 4 in Fig. 13. The estimation is similar for both models at $M_{0}=2$ and close to Eulerian simulations (Fig. 13-a), while GSD seems better for strong shocks (Fig. 13-b).

In Fig. 14, we compare the angle of the shock-shock trajectory $\chi$ for different angles $\theta_{w}$ at initial Mach number equal to 1.5 (Fig. 14-a) and 10 (Fig. 14b). The three-shock theory [3] is also considered for the strong shock case as suggested by Whitham [36]. For a weak shock (Fig. 14-a), both models are similar due to the close velocity of the waves. The estimation of the shock front 
is well estimated. The models are in good agreement with Eulerian simulations when a triple point exists. Physically, for some configurations $\left(M_{0}, \theta_{w}\right)$, in the von Neumann reflection regime [3] for example, the triple point does not exist. The transition between the Mach stem and the incident shock is then continuous, but with the GSD and KIN models a triple point is always present. For the strong shock case (Fig. 14-b), the shock-shock moves faster for GSD than for KIN as expected from the wave velocity analysis. The behavior of both models is very distinct. For a small deflection angle $\left(\theta_{w}<20^{\circ}\right)$, the Kinematic approach is close to the three-shock theory and Eulerian data, while for a higher angle $\left(\theta_{w}>40^{\circ}\right)$ Whitham's approach seems better. In any event, considering the simplicity of these models, one can observe a good agreement with data.

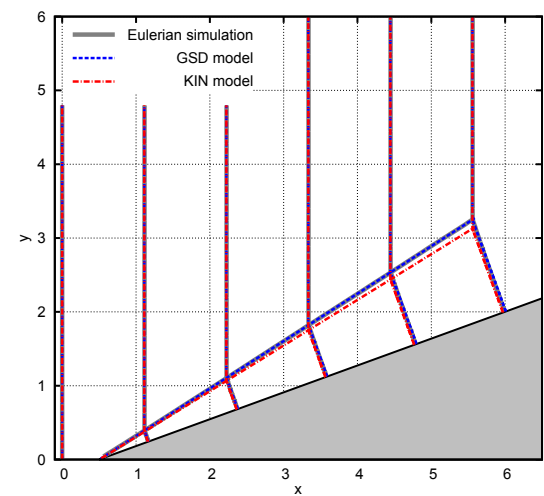

(a)

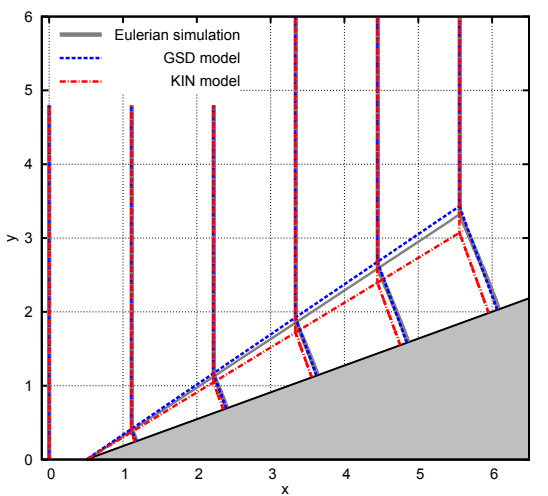

(b)

Figure 12: Diffraction of a planar shock over a concave corner of angle 20 degrees at Mach numbers 2 (a) and 4 (b). The shock travels from left to right. Comparison of shock positions and shock-shock trajectory between the GSD and KIN models with Eulerian simulation. Shock-shock trajectory is superimposed for GSD model and Eulerian simulation in Fig. (a).

\subsection{Numerical solutions}

We now consider some more complicated problems requiring the use of the $2 \mathrm{D}$ conservative Lagrangian scheme briefly described in section 3.3 to exhibit a solution for the GSD and KIN models.

Diffraction over a cylinder or a sphere. Let us consider the diffraction of a planar shock over a cylinder. During the propagation of the shock, two shockshocks develop on the front. The first one appears when the shock diffracts over the cylinder, and the second one occurs once the front interacts with the axis of symmetry. We present the solution obtained at Mach number 2.81 in Fig. 15-a. Successive shock positions are plotted, as well as the shock-shock trajectories. It 


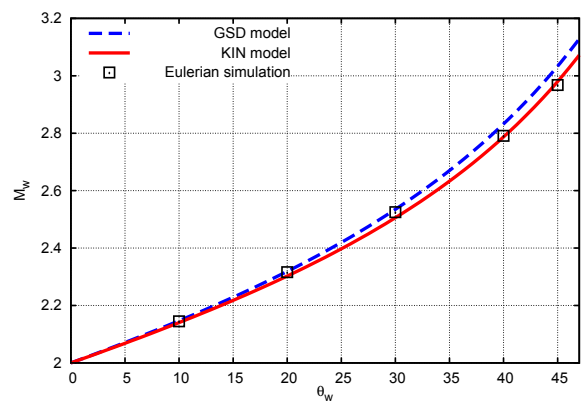

(a)

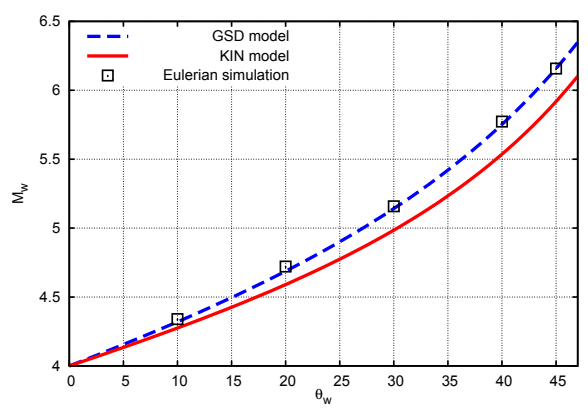

(b)

Figure 13: Wall Mach number $M_{w}$ with respect to deflection angle $\theta_{w}$, in degree, for diffraction of a planar shock over concave corner at Mach numbers 2 (a) and 4 (b).

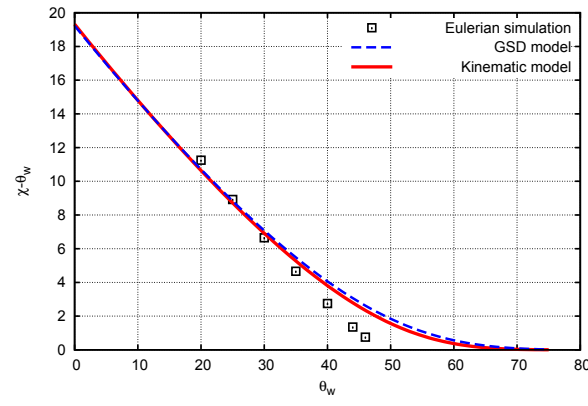

(a)



(b)

Figure 14: Shock-shock trajectory $\chi-\theta_{w}$ with respect to deflection angle $\theta_{w}$, in degree, for diffraction of a planar shock over a concave corner at Mach numbers 1.5 (a) and 10 (b). Comparison of the GSD and KIN models with the threeshock theory from [3] and Eulerian simulations. 
is worth noticing the good agreement of these trajectories with the experimental data, obtained from [36], and with the Eulerian simulation results. Surprisingly, note the better estimation for the first triple point with the KIN model.

Fig. 15-b gives the solution for the diffraction over a sphere at Mach number 2.85. The numerical results are obtained in a $2 \mathrm{D}$ axisymmetric approximation. Here again, one can see that both models estimate the shock-shock trajectories well, with a slightly better estimation of the first shock-shock for the Kinematic model. Note that in this case, experimental shock-shock trajectories are very close for Mach numbers 2.85 and 4.41 as observed by Henshaw et al. [13]

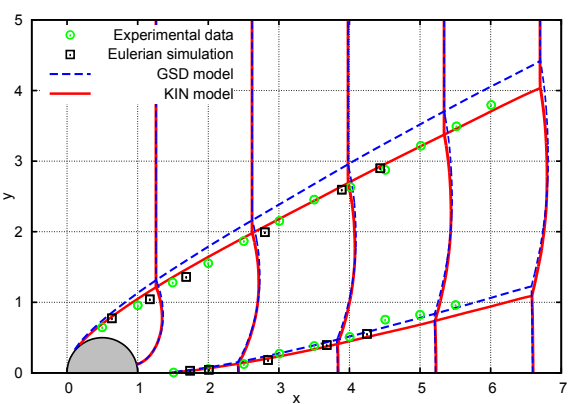

(a)

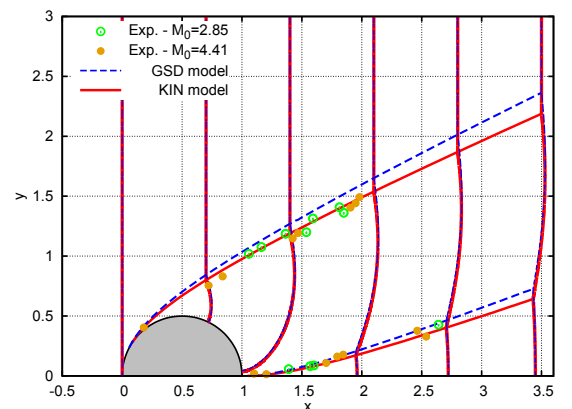

(b)

Figure 15: Diffraction of a planar shock over a cylinder at Mach number 2.81 (a) and over a sphere at Mach number 2.85 (b) (2D axi-symetrical simulation). The shock travels from left to right. Comparison of shock-shock trajectories between the GSD and KIN models with experimental data from [36] and Eulerian simulation. $(O x)$ axis is the symmetry line.

Diffraction over a mound. The next example is the planar diffraction over a mound with a perfectly reflecting ground. Like in the previous case, two shock-shocks form on the front: the first one when the shock interacts with the mound, and the second one when it diffracts over the ground. Results for $M_{0}=2$ and $M_{0}=4$ are presented in Fig. 16. At Mach number 2 (Fig. 16-a), the shock positions for the GSD and KIN models are close to one another and rapidly overtake the front location from Eulerian simulations. The trajectories of the second shock-shock for GSD and KIN are much closer to one another than the first ones. Regarding the first trajectory, the Kinematic model approaches Euler results better. At Mach number 4, both models exhibit significant differences. They are still overtaking the shock front positions compared to Eulerian simulations (Fig. 16-b). There is a visible difference between the shock-shock trajectories, and the KIN model is again closer to the Eulerian simulation.

Diffraction over a trough. Finally, let us consider the planar diffraction over a trough. It is somehow the mirror problem of the previous case. Indeed 




(a)

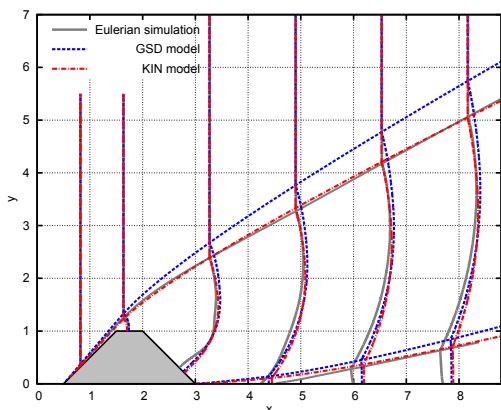

(b)

Figure 16: Diffraction of a planar shock over a mound at Mach numbers 2 (a) and 4 (b). The shock travels from left to right. Comparison of shock positions and shock-shock trajectories between the GSD and KIN models with Eulerian simulation. The ground is located at $y=0$.

in the last example, the shock is first compressive (a shock-shock appears), then expansive, and at last compressive. In this example, the shock is successively expansive, compressive and expansive. Only one shock-shock occurs here. The GSD and KIN models are compared with the Eulerian simulation results at Mach numbers 2 (Fig. 17-a) and 4 (Fig. 17-b). Both models overestimate the positions of the shock, as well as the shock-shock trajectory. The KIN model is slightly better, for this particular configuration, but nevertheless displays large differences with the Eulerian simulation.

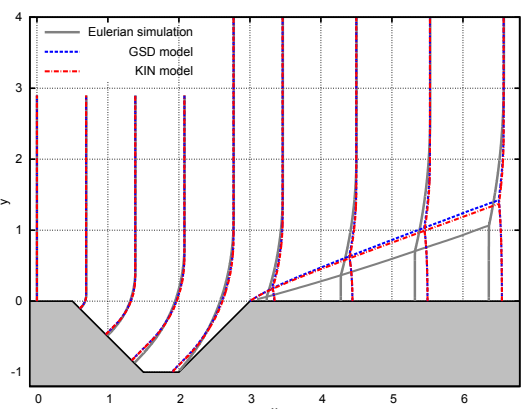

(a)

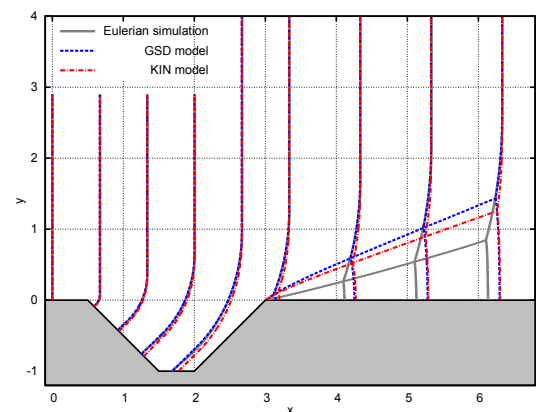

(b)

Figure 17: Diffraction of a planar shock over a trough at Mach numbers 2 (a) and 4 (b). The shock travels from left to right. Comparison of shock positions and shock-shock trajectory between the GSD and KIN models with Eulerian simulation results. The ground is located at $y=0$. 
Synthesis. For the range of configurations tested, the GSD model is closer to experimental data and Eulerian simulation than the KIN model for elementary Riemann problems, namely problems with only expansion or compression waves. Despite this observation, KIN turns out to be slightly better for problems combining these two waves, mainly due to error counterbalance. In regions where the shock is expansive, the shock position is overestimated by the KIN model, and when the shock is compressive, the shock-shock is slower for the KIN approach, implying also a lower Mach stem. The GSD model globally tends to overestimate the shock-shock position.

\section{Conclusion and perspectives}

In this paper, the Kinematic model was studied and compared with the GSD model. Both models are simplified hyperbolic systems for shock wave propagation modeling only the leading front. Although the mathematical derivations of each approach are different, the structural equivalence of the models has been proven. It is indeed natural to assume that the shock front evolves along ray tubes. The Kinematic model has thus been reformulated under the same form as GSD, but with its own $A-M$ relation. This equivalence between the models allowed a complete study of the Kinematic approach, as well as an easy comparison with the GSD model. In 2D, the study of elementary Riemann problems has shown that the shock travels faster for KIN than for GSD when the shock is expanding, while shock-shocks are slower for KIN. These differences tend to disappear when the shock intensity decreases, that is for Mach numbers lower than 2. For cases with only expansion or compression waves, GSD is better than the KIN model. For more complex problems combining these two types of waves, like diffraction over a cylinder or a mound, the KIN model seems slightly better, at least for the problems tested in this paper. Considering the simplicity of these models, the results are satisfactory in comparison to experimental and numerical data.

However, both models suffer from the same limitation for the diffraction over a convex wall. For sufficiently weak shocks, no solution exists above a given deflection angle. This could be related to the underlying hypotheses of the models. Some important information are lost for weak shocks considering only the leading front and neglecting post-shock flow. This issue could be addressed in further investigations.

\section{Acknowledgement}

Part of this work has been possible thanks to the LETMA collaboration: a contractual research laboratory between CEA, CNRS, École Centrale Lyon, CInnov and Université Pierre et Marie Curie. O. Gainville is acknowledged for valuable discussions. 


\section{References}

[1] R. Arora and M. J. Siddiqui. Evolutionary behavior of weak shocks in a non-ideal gas. Journal of Theorical and Applied Physics, 7(1):1-6, 2013.

[2] S. Baskar and P. Prasad. Propagation of curved shock fronts using shock ray theory and comparison with other theories. Journal of Fluid Mechanics, 523:171-198, 2005.

[3] G. Ben-Dor. Shock wave reflection phenomena, volume 2. Springer, 2007.

[4] C. Besset and E. Blanc. Propagation of vertical shock waves in the atmosphere. The Journal of the Acoustical Society of America, 95(4):1830-1839, 1994.

[5] J. P. Best. A generalisation of the theory of geometrical shock dynamics. Shock Waves, 1(4):251-273, 1991.

[6] C. Catherasoo and B. Sturtevant. Shock dynamics in non-uniform media. Journal of Fluid Mechanics, 127:539-561, 1983.

[7] W. Chester. The quasi-cylindrical shock tube. The London, Edinburgh, and Dublin Philosophical Magazine and Journal of Science, 45(371):1293-1301, 1954.

[8] W. Chester. The propagation of shock wave along ducts. Advances in Applied Mechanics, 6:119-152, 1960.

[9] R. Chisnell. The motion of shock wave in a channel, with applications to cylindrical and spherical shock waves. Journal of Fluid Mechanics, 2(3):286-298, 1957.

[10] R. Collins and H.-T. Chen. Motion of a shock wave through a nonuniform fluid. In Proceedings of the Second International Conference on Numerical Methods in Fluid Dynamics, pages 264-269. Springer, 1971.

[11] R. Courant and K. O. Friedrichs. Supersonic flow and shock waves, volume 21. Springer Science \& Business Media, 1999.

[12] S. Éveillard, N. Lardjane, J.-Y. Vinçont, and I. Sochet. Towards a fast-running method for blast-wave mitigation by a prismatic blast wall. Comptes Rendus Mécanique, 341(8):625 - 635, 2013.

[13] W. Henshaw, N. Smyth, and D. Schwendeman. Numerical shock propagation using geometrical shock dynamics. Journal of Fluid Mechanics, 171:519-545, 1986.

[14] H. T. Huynh. Accurate monotone cubic interpolation. SIAM Journal on Numerical Analysis, 30(1):57-100, 1993. 
[15] H. Jourdren. HERA: a hydrodynamic AMR platform for multi-physics simulations. In Adaptive Mesh Refinement-Theory and Applications, pages 283-294. Springer, 2005.

[16] W. E. Lorensen and H. E. Cline. Marching cubes: A high resolution 3d surface construction algorithm. In ACM siggraph computer graphics, volume 21, pages 163-169. ACM, 1987.

[17] M. Nguyen-Dinh, N. Lardjane, C. Duchenne, and O. Gainville. Direct simulations of outdoor blast wave propagation from source to receiver. Shock Waves, pages $1-22,2017$.

[18] Y. Noumir, A. Le Guilcher, N. Lardjane, R. Monneau, and A. Sarrazin. A fast-marching like algorithm for geometrical shock dynamics. Journal of Computational Physics, 284:206-229, 2015.

[19] K. Oshima, K. Sugaya, M. Yamamoto, and T. Totoki. Diffraction of a plane shock wave around a corner. ISAS report, 30(2):51-82, 1965.

[20] M. Pandey and V. Sharma. Kinematics of a shock wave of arbitrary strength in a non-ideal gas. Quarterly of Applied Mathematics, 67(3):401418, 2009.

[21] D. Schwendeman. A numerical scheme for shock propagation in three dimensions. In Proceedings of the Royal Society of London A: Mathematical, Physical and Engineering Sciences, volume 416, pages 179-198, 1988.

[22] D. Schwendeman. A new numerical method for shock wave propagation based on geometrical shock dynamics. In Proceedings of the Royal Society of London A: Mathematical, Physical and Engineering Sciences, volume 441, pages 331-341, 1993.

[23] D. Schwendeman and G. Whitham. On converging shock waves. In Proceedings of the Royal Society of London A: Mathematical, Physical and Engineering Sciences, volume 413, pages 297-311, 1987.

[24] D. W. Schwendeman. A higher-order Godunov method for the hyperbolic equations modelling shock dynamics. In Proceedings of the Royal Society of London A: Mathematical, Physical and Engineering Sciences, volume 455, pages 1215-1233, 1999.

[25] V. Sharma and C. Radha. On one-dimensional planar and nonplanar shock waves in a relaxing gas. Physics of Fluids, 6(6):2177-2190, 1994.

[26] V. Sharma and C. Radha. Three dimensional shock wave propagation in an ideal gas. International Journal of Non-Linear Mechanics, 30(3):305-322, 1995.

[27] V. Sharma and R. Venkatraman. Evolution of weak shocks in one dimensional planar and non-planar gasdynamics flows. International Journal of Non-Linear Mechanics, 47(8):918-926, 2012. 
[28] B. W. Skews. The shape of a diffracting shock wave. Journal of Fluid Mechanics, 29(02):297-304, 1967.

[29] C. Truesdell and R. Toupin. The classical field theories. In Principles of Classical Mechanics and Field Theory/Prinzipien der Klassischen Mechanik und Feldtheorie, pages 226-858. Springer, 1960.

[30] P. A. Varadarajan. Noise transmission along shock-waves. PhD thesis, The University of Michigan, 2011.

[31] G. Whitham. A new approach to problems of shock dynamics. Part I: Twodimensional problems. Journal of Fluid Mechanics, 2(2):145-171, 1957.

[32] G. Whitham. On the propagation of shock waves through regions of nonuniform area or flow. Journal of Fluid Mechanics, 4(04):337-360, 1958.

[33] G. Whitham. A new approach to problems of shock dynamics. Part II: Three-dimensional problems. Journal of Fluid Mechanics, 5(3):369-386, 1959.

[34] G. Whitham. A note on shock dynamics relative to a moving frame. Journal of Fluid Mechanics, 31(03):449-453, 1968.

[35] G. Whitham. Gas dynamics. In Linear and Nonlinear Waves, chapter 6. John Wiley \& Sons, Inc., 3rd edition, 1999.

[36] G. Whitham. Shock dynamics. In Linear and Nonlinear Waves, chapter 8. John Wiley \& Sons, Inc., 3rd edition, 1999.

[37] T. W. Wright. An intrinsic description of unsteady shock waves. The Quarterly Journal of Mechanics and Applied Mathematics, 29(3):311-324, 1976.

[38] H. Zhao-Yuan and Y. Xie-Zhen. Geometrical shock dynamics. In Handbook of Shock Waves, volume 1, chapter 3.7, pages 485-550. Academic Press, 2000 . 